\title{
Autonomous Selection and Printing of 3D Models for People Who Are Blind
}

\author{
T. GÖTZELMANN, Nuremberg Institute of Technology
}

3D models are an important means for understanding spatial contexts. Today these models can be materialized by 3D printing, which is increasingly used at schools for people with visual impairments. In contrast to sighted people, people with visual impairments have so far, however, neither been able to search nor to print 3D models without assistance. This article describes our work to develop an aid for people with visual impairments that would facilitate autonomous searching for and printing of 3D models. In our initial study, we determined the requirements to accomplish this task by means of a questionnaire and developed a first approach that allowed personal computer-based 3D printing. An extended approach allowed searching and printing using common smartphones. In our architecture, technical details of 3D printers are abstracted by a separate component that can be accessed via Wi-Fi independently of the actual 3D printer used. It comprises a search of the models in an annotated database and 3D model retrieval from the internet. The whole process can be controlled by voice interaction. The feasibility of autonomous 3D printing for people with visual impairments is shown with a first user study. Our second user study examines the usability of the user interface when searching for 3D models on the internet and preparing them for the materialization. The participants were able to define important printing settings, whereas other printing parameters could be determined algorithmically.

CCS Concepts: • Human-centered computing $\rightarrow$ Accessibility systems and tools; Auditory feedback; Ubiquitous and mobile devices;

Additional Key Words and Phrases: 3D printing, autonomous, independent, self-reliant, tactile, graphics, 3D models, internet, retrieval, print server, hardware abstraction, visually impaired, blind, accessibility, tangible, exploration, user study

\section{ACM Reference format:}

T. Götzelmann. 2018. Autonomous Selection and Printing of 3D Models for People Who Are Blind. ACM Trans. Access. Comput. 11, 3, Article 14 (September 2018), 25 pages.

https://doi.org/10.1145/3241066

\section{INTRODUCTION}

Several spatial objects for tactile exploration can be found in schools for people with visual impairments, in large libraries and highly frequented places. These objects enable people to experience 3D objects using the tactile sense and to understand spatial structures and relations.

3D model search and printing can used to create these spatial objects [8], but it is not generally accessible. Unlike for sighted people, it is not a trivial task for people who are blind to search and

\footnotetext{
Authors' address: T. Götzelmann, Nuremberg Institute of Technology, Keßlerplatz 12, D-90489 Nürnberg; email: Timo.Goetzelmann@ohm-university.org.

Permission to make digital or hard copies of all or part of this work for personal or classroom use is granted without fee provided that copies are not made or distributed for profit or commercial advantage and that copies bear this notice and the full citation on the first page. Copyrights for components of this work owned by others than ACM must be honored. Abstracting with credit is permitted. To copy otherwise, or republish, to post on servers or to redistribute to lists, requires prior specific permission and/or a fee. Request permissions from permissions@acm.org. (C) 2018 Association for Computing Machinery. 1936-7228/2018/09-ART14 \$15.00 https://doi.org/10.1145/3241066
} 
find interesting models of 3D objects on the internet. To obtain a first spatial impression of 3D objects, for sighted people it is often sufficient to view images and illustrations. If images from different viewing perspectives are not enough, sighted people can access online 3D viewing services, potentially using 3D glasses, to get better comprehension. The models can also be materialized by means of 3D printers to make them perceivable by the tactile sense. For people with visual impairments, this process is largely inaccessible.

The barriers begin with the search for spatial models on the internet. The broad range of free 3D models remains reserved to sighted people because the search results are presented visually. Furthermore, without sight, people face additional issues with the realization of the print job since there are no accessible tools for the processing and print preparation of $3 \mathrm{D}$ models $[6,7]$. The operation of 3D printers themselves presents several barriers. The postprocessing of the print results also presents challenges for people who are blind. This starts when the object has to be removed from the building plate. In addition, supporting structures necessary for correct printing represents a further obstacle, as they have to be removed manually after printing. For people who are blind, it is not obvious which parts of the model have to be removed. The removal itself is also problematic, as the parts often have to be cut off with gloves, protective goggles, and pliers to prevent injuries.

Many schools for special education already possess off-the-shelf 3D printers. Individual models can be printed with the help of assistants. However, this strategy does not respect the informal self-determination and privacy of people who are blind. While there are several approaches that aim to partially automate 3D printing, they focus mainly on creating 3D materials. In the schools mentioned above, children should be encouraged to participate in special training for using 3D printers [7]. However, the interfaces have to be made more accessible to them. This article pursues this goal and aims at improving the autonomy of people with visual impairments, beginning with the retrieval of spatial models. It focuses on people who are blind. However, because this development could be advantageous for people with residual vision, in the following we will use the more general term, people with visual impairments.

This article is an expanded version of a short paper [15] accepted at the $10^{\text {th }}$ International ACM Conference on Pervasive Technologies Related to Assistive Environments. The short paper presented a monolithic approach based on a prototypical personal computer configuration. This setup was used to test the principle feasibility of the technical 3D printing process for people who are blind. In contrast, the novel contributions of this paper include a flexible architecture that allows 3D printer hardware abstraction. Ordinary smartphones can be used as an interface for the entire printing process. This includes the initial search process, the print preparation, and the taskspecific control of the 3D printer. For searching the models, it combines the use of an annotated 3D model database and the retrieval of 3D models from the internet. The user requirements for the created concept were collected with a questionnaire. Finally, the approach was evaluated through a comprehensive user study with people with visual impairments.

\section{RELATED WORK}

This section reports the state-of-the art of works that enable people with visual impairments (VIP) to retrieve documents such as 3D models from the internet. In addition, it discusses approaches that address the automated creation of tactile materials and their physical representations by 3D printing.

\subsection{Retrieval of Web Content for People Who Are Blind}

The accessibility to many documents on the web is rather limited for VIP [21, 34, 39]. The search process itself has been addressed by multiple researchers. The subject matter of several studies (e.g., References [2, 33, 43]) for people with visual impairments was the usability of existing 
search engines to retrieve HTML web pages. The approach BrookesTalk [58] used multiple voices to improve the retrieval process of web pages. However, these approaches mainly relied on the adaption of two-dimensional visual information into sequential verbal information that can be read by VIP using tools such as screen readers.

To preserve the spatial characteristic of the webpages' visual layout other approaches provided auditory interfaces for the exploration of web content. Goose and Möller [14] mapped the structure of HTML documents into a 3D audio space and explored this space through audio signals. The approach Websound [40] transformed structural components of web pages into spatial auditory representations that could be explored by an audio-haptic interface and combined it with text-to-speech output for textual components. A subsequent multimodal approach introduced the sonification of web pages for VIP [41], including text and images. Another approach for audiohaptic retrieval of web information was presented by Yu et al. [57]. This article investigated the use of a force feedback mouse for the exploration of web pages by a user study. A different approach mapped web pages onto 3D virtual environments [26]. Users who were blind were able to perceive these virtual environments by the haptic sense and thus perceive the structure of the web page.

Besides the improvement of accessibility of web pages for VIP, other approaches addressed accessibility enhancement for spatial information. An analysis by White et al. [55] investigated the accessibility of 3D virtual online environments such as Second Life, for people who are blind. TextSL [11] represented a text interface for virtual worlds in which users were able to use commands to navigate. An approach by Nemec et al. [37] enabled users with visual impairments to navigate inside a virtual environment and to query for objects within the 3D scene while walking through it.

However, to our knowledge, there is no approach that is designed to facilitate the access to 3D model retrieval engines. The majority of these search engines use textual input to retrieve tagged 3D models. However, their search results are presented in a highly visual manner, i.e., depictions of 3D models. In some cases, these result sets are combined with extensive descriptions. This work addresses these issues by providing an accessible interface for 3D model searching, selection, and download.

\subsection{Automated Creation of Tactile Content for People Who are Blind}

Several approaches were designed to allow sighted people to produce tactile material for people with visual impairments (e.g., for teaching materials). Some approaches addressed the translation of images [54] to their tactile representation and the efficiency [32] of generating the translations to print the results with established tactile printing technologies. Others focused on the generation of 3D print content, such as tactile data visualizations [5, 27], detail maps [17, 47], movable tactile pictures for children [29], print, accessible interfaces [20] for common electronic devices through a transparent tactile layer, and acoustic markers [44] for 3D objects that are complemented with audio labels. In contrast to these approaches, the following paragraph describes works that aimed at enabling VIP to autonomously create print content.

Multiple approaches automatically transcribed digital images into tactile representations. Wang et al. [51] proposed an automatic system using geometric abstraction and optical character recognition to transform map scans into tactile graphics. Another approach [48] achieved this from hand-drawn images. Other approaches directly generated tactile graphics from logical data such as geographical descriptions. Gual et al. [19] proposed to integrate volumetric tactile symbols into 3D printed tactile maps.

\subsection{Autonomous Creation of Tactile Content by People with Visual Impairments}

To increase the autonomy of people with visual impairments, TMACS [52] generated tactile maps along a user defined route. The feasibility of autonomous tactile map creation has been shown 
by Watanabe et al. [52]. Other map-related approaches added interactive components [16] and customization [49]. BlindWeb Maps [17] introduced an accessible web-service that generated tactile maps for 3D printers and other tactile printing technologies. An accessible web page enabled users who were blind to autonomously generate tactile graphs from numerical data [53]. In a project of Yahoo Japan [23], a machine was built that allowed children to choose between a limited set of locally stored 3D models using voice input. The machine subsequently started printing. However, the further steps were inaccessible (e.g., print removal, elimination of support structures) and the database was limited to a predefined set of 3D models. The small number of configuration options, the proprietary architecture, and the missing abstraction of the printing hardware impeded its dissemination to individuals with visual impairments.

Currently, there is no open source 3D modelling software available that is accessible to people who are blind [6]. Furthermore, there is no approach that assists users who are blind in the autonomous preparation of 3D prints and during the actual printing process. In the following, we introduce an approach that combines both the autonomous retrieval as well as the printing of 3D models in a form that is accessible to people who are blind.

\subsection{Requirements Analysis by Questionnaire}

This section reports on a questionnaire we administered to determine the requirements of people who are blind for an application that would retrieve and print 3D models.

2.4.1 Participants and Apparatus. The questionnaire was completed by 52 individuals who were on average $44.16 \pm 12.65$ years old. Besides questions concerning demographic variables, it consisted of eight questions to determine the requirements for our approach (see Appendix A). The URL of our web-based questionnaire (Survey Monkey) was spread by a popular national newsletter for people with visual impairments.

2.4.2 Design and Procedure. The first four questions were grouped and aimed to determine whether an application that allows autonomous retrieval and printing of 3D models is desirable for VIP. The first question requested the subjects to specify how many 3D objects they have used to date to understand spatial facts (i.e., models in schools, touchable building models, etc.). This was recorded as a categorical variable with the parameter values $0-10,11-50,51-100$, and $>100$. The following three questions were recorded as binary variables. The users were asked:

- Would they consider $3 D$ printing at home favorable?

- Would it be advantageous if 3D models could be printed without assistance of a sighted user?

-If autonomous $3 D$ printing would be possible, would they invest in an suitable $3 D$ printer for an estimated price of 800 Euros?

The second block consisted of multiple individual questions about the requirements for the user interface. One question concerned the basic willingness of the participants to install an appropriate smartphone application. Another question concerned the disposition of the participants to participate actively in the discussion about models and in the sharing process for already printed 3D models. A multiple choice question concerned the importance that participants assigned to categories of 3D models (technical models, architectural models, art, nature, and persons) that participants assigned to them. The participants were encouraged to write down their own suggestions.

Another multiple choice question included a Likert scale consisting of four items. This scale was used to determine the self-assessed needs of the target group to adjust printing parameters of selected 3D models (printing size, printing quality, printing duration, and the stability of prints). Each item consisted of five categories (unimportant, slightly unimportant, neutral, slightly important, important), which were encoded in the range [1..5]. Besides the predefined options, the Likert scales included an additional text field that allowed respondents to propose own ideas. 


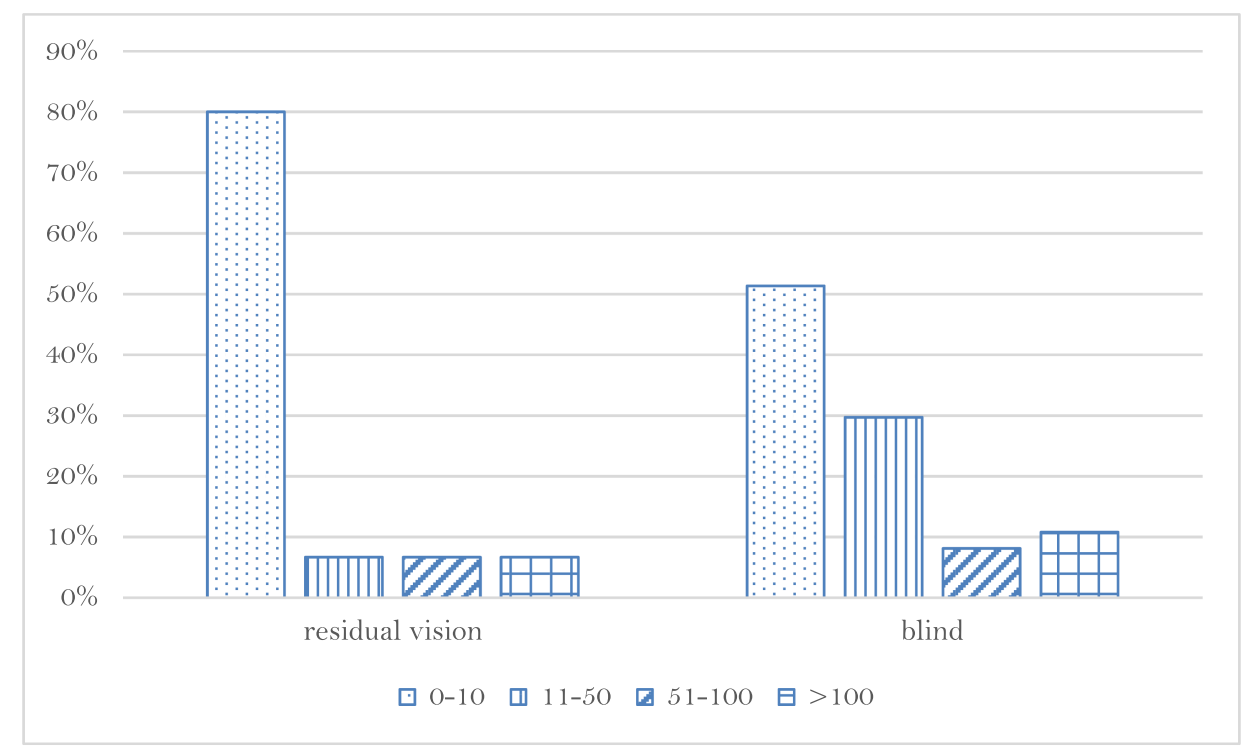

Fig. 1. Most of the participants only had limited access to models for the tactile exploration of spatial objects.

2.4.3 Results. On average, the users spent $9.08 \pm 7.16$ minutes on the survey. Since it was not compulsory to complete every question, only 49 users answered every question. Thirty-seven of the participants reported they were blind, accordingly placing them in WHO category 4/5. Fifteen participants had residual vision between 2 and $10 \%$ (WHO category 2/3).

The first block of questions was answered in full by each participant. The first question, "How many 3D models have you used so far for tactile exploration (e.g., tactile models at school, building models, animal or machine models)?" showed evidence that most of the participants only had the opportunity to use very few 3D models of spatial objects for exploration. People who are blind tended to make more usage of the exploration of spatial models (see Figure 1). However, the difference was not significant between people with residual vision $(>2 \%)$ and people who are blind $\left(\chi^{2}(3)=2.61, p=.46\right)$.

The great majority $(84.62 \%)$ of participants indicated that the 3D printing of objects would be desirable for them (Question 2). 90.38\% of the participants mentioned that it would have an additional benefit if they could select and print these 3D models without the assistance of a sighted person (Question 3). Finally, 65.38\% of the participants would be willing to buy an suitable 3D printer for 800 Euros (Question 4).

In the second block, $92.31 \%$ agreed that they would install a dedicated mobile application on their smartphone (Question 6). 84.62\% of the participants agreed that they would actively participate in the discussion about already printed 3D models and share them amongst other VIP (Question 7). They ranked their interest in the provided five categories of 3D models (Question 5) in the following order:

- Technical models (Machines, ...) 
Table 1. Distribution of Users' Answers about the Importance of the Ability to Adjust the Following Parameters for 3D Printing by a Mobile Application

\begin{tabular}{|c|c|c|c|c|c|}
\hline & unimportant & $\begin{array}{c}\text { slightly } \\
\text { unimportant }\end{array}$ & neutral & $\begin{array}{l}\text { slightly } \\
\text { important }\end{array}$ & important \\
\hline $\begin{array}{l}\text { Printing } \\
\text { size }\end{array}$ & $\begin{array}{c}1 \\
(1.96 \%)\end{array}$ & $\begin{array}{c}2 \\
(3.92 \%)\end{array}$ & $\begin{array}{c}26 \\
(50.98 \%)\end{array}$ & $\begin{array}{c}5 \\
(9.80 \%)\end{array}$ & $\begin{array}{c}17 \\
(33.33 \%)\end{array}$ \\
\hline $\begin{array}{l}\text { Printing } \\
\text { quality }\end{array}$ & $\begin{array}{c}1 \\
(1.96 \%)\end{array}$ & $\begin{array}{c}2 \\
(3.92 \%)\end{array}$ & $\begin{array}{c}23 \\
(45.10 \%)\end{array}$ & $\begin{array}{c}11 \\
(21.57 \%)\end{array}$ & $\begin{array}{c}14 \\
(27.45 \%)\end{array}$ \\
\hline $\begin{array}{l}\text { Printing } \\
\text { time }\end{array}$ & $\begin{array}{c}10 \\
(19.61 \%)\end{array}$ & $\begin{array}{c}9 \\
(17.65 \%)\end{array}$ & $\begin{array}{c}21 \\
(41.18 \%)\end{array}$ & $\begin{array}{c}6 \\
(11.76 \%)\end{array}$ & $\begin{array}{c}5 \\
(9.80 \%)\end{array}$ \\
\hline $\begin{array}{l}\text { Stability } \\
\text { of print }\end{array}$ & $\begin{array}{c}6 \\
(11.76 \%)\end{array}$ & $\begin{array}{c}3 \\
(5.88 \%)\end{array}$ & $\begin{array}{c}12 \\
(23.53 \%)\end{array}$ & $\begin{array}{c}13 \\
(25.49 \%)\end{array}$ & $\begin{array}{c}17 \\
(33.33 \%)\end{array}$ \\
\hline
\end{tabular}

To our surprise, the participants made extensive use of the free text field and provided many examples. We aggregated their ideas into these additional categories:

-Functional models (Tools, spare parts, ...)

11 entries

- Maps and topographic models (Hills, street maps, ...)

10 entries

- Mathematical and scientific models (Curves, crystals, molecules, ...)

5 entries

- Anatomical models (Bones, heart, ...)

- Signs and symbols (Characters, logos, ...)

3 entries

3 entries

Finally, the participants had to decide how important they considered four predefined features of the mobile application to adjust printing parameters (Question 8). Fifty-one participants completed this question. Overall, multiple participants had a considerable desire to adjust multiple printing parameters before they would start the printing process. A Wilcoxon Signed Rank Test indicated that the printing size $(Z=1265.5, p<.0)$, printing quality $(Z=1268.5, p<.0)$ and stability $(Z=1152.0, p<.0)$ of the print significantly differed from the neutral position. The most subjects preferred to adjust parameters (see Table 1) for the size of the printing object (ø: $3.69 \pm$ 1.04 ) and its quality (ø: $3.69 \pm 0.98)$. The ability to adjust the stability of $3 \mathrm{D}$ prints was also considered as important (ø: $3.63 \pm 1.31$ ).

The printing time showed a tendency to be unimportant to the majority of subjects (ø: $2.25 \pm$ 1.19). However, the difference of printing time compared to the neutral position was statistically insignificant $(Z=801.5, p=.181)$.

2.4.4 Discussion. The study received a good response and much positive feedback. However, it cannot be ruled out that a large number of participants with a positive attitude toward new technologies such as the internet and 3D printing took part. As expected, only a few people who are blind had the previous experience of exploring spatial facts using tactile 3D models. This is probably due to the fact that there are simply few resources available for this purpose and to this target group. This was even more evident for people with residual vision, which is probably due to the fact that they prefer visual stimuli. However, it cannot be excluded that there are countryspecific differences.

The large majority of participants were quite positive about the possibility of printing 3D models on their own. Ideally, they would do this without the need for a sighted assistant and more than half of the respondents could imagine buying their own 3D printer. The vast majority also agreed to install a corresponding application on their smartphone. 


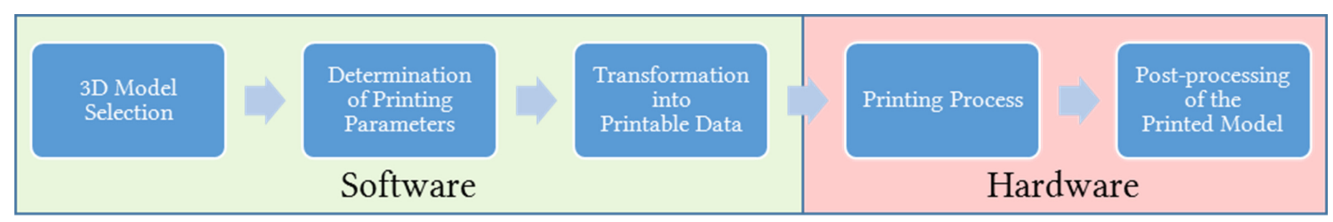

Fig. 2. Steps of the 3D printing process and their assignment to software and hardware.

Many of the participants would not only use the tool for themselves, but would also share their experiences with the printed 3D models with others. In this way, a structured database could be created, which, in addition to the models, would contain information from people who are blind (optimal printing size, correspondence between description and actual model, detail how to explore tactile model, etc.), which could help other users to select and print models. A corresponding functionality is supposed to be integrated into the resulting application. On the one hand, the variety of 3D models is to be made possible by information retrieval methods in large 3D servers, and on the other hand, a structured and a database, annotated by people who are blind, with models suitable for 3D printing should be created.

The given categories of printed objects were considered meaningful by many participants. However, numerous other categories were mentioned that also should be included in a structured database.

The controllability of print size and quality was important to most participants. On the other hand, adjustability of the printing time was less important. In addition to these features, however, stability was important to many. Since the stability of nonmechanically stressed models can easily be set as a standard, they will not be included in user-determined settings. In contrast to this, the print size and quality is queried before printing, while the user is informed of the resulting print time.

\section{APPROACH}

In this section, we introduce an approach for the autonomous selection and printing of 3D models. Based on the questionnaire and informal conversations with people of the target group, we developed several requirements. The approach should not only enable VIP to print 3D models on their own, but should also allow the selection of 3D models. The search process itself should be assisted by verbal descriptions incorporating technical details about the 3D models without the need of a sighted assistant. Before the selected model is printed, users who are blind should have the opportunity to configure important printing parameters. After printing is finished, VIP should be able to retrieve the finished product and to remove support structures without assistance. Finally, the approach should be developed in a generic way using affordable, existing technologies.

Beginning from the selection of existing 3D models, several tasks have to be performed before the finished 3D model can be used for tactile exploration. In the following, we broke down this task into multiple subsequent steps (see Figure 2) and adapted them to the needs of VIP. Some of these adaptations solely apply to software, others mainly concern the hardware, including the printed results.

\subsection{D Model Selection}

For sighted users, millions of free 3D models are available on the internet that can be easily downloaded and printed at home. Search engines exist that can be used by text retrieval (e.g., Reference [12]) or by geometric similarity (e.g., Reference [46]). An alternative approach is to browse through categories of websites hosting or linking 3D models (e.g., References [45, 50, 56]). 


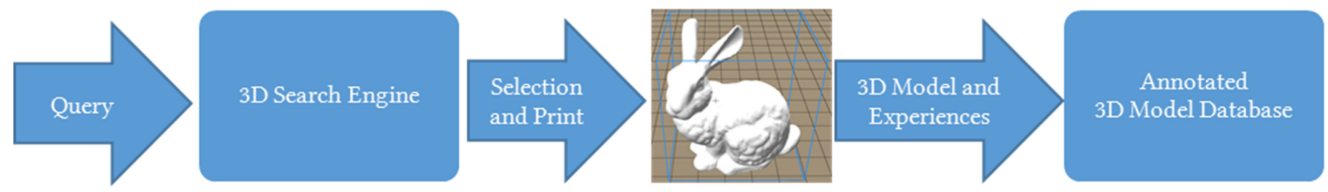

Fig. 3. Retrieval of 3D models by search engines and construction of a continually expanding database for printed models and experiences of VIP.

However, these categories do not primarily address the needs of VIP. As reported in Section 2.1, there are multiple approaches to make web resources accessible to people with visual impairments. However, there is no accessible approach for retrieving 3D models from the internet. Hence, it would be useful to allow VIP to benefit (i) from the variety of models accessible by text retrieval as well as (ii) from the structured organization of categories tailored to the needs of people who are blind.

For model selection, we propose the following concept: users should be able to access a wide variety of 3D models by information retrieval. To correspond to the claim of Reference [7] and also to enable children easy access to 3D printing, this should be supported by speech recognition. However, some of the models retrieved and their descriptions may not be suitable for autonomous printing for VIP. According to Reference [8], only approximately $30 \%$ of the models require no assembly. Hence, 3D objects that must be manually assembled from multiple parts will be filtered out. Based on the results of the questionnaire (see Section 2.4.3), experience with already printed models (e.g., optimal print size, alternative description) should be shared in a categorized 3D model database (see Figure 3). The structure of the database will be based on the results of questionnaire.

\subsection{Determination of Printing Parameters}

The most common consumer 3D printing technology is Fused Filament Fabrication (FFF). In FFF, the object is created layer by layer through the deposit of molten plastic. Additional printing material can be deposited on already solidified layers. For upward laterally extending structures (e.g., treetops), however, it is sometimes necessary to print support structures, since in this case it is not possible to build on previously printed lower layers. These support structures have to be removed after the print process is finished.

Before the actual print process starts, the desired 3D model has to be prepared for printing. User interfaces for the preparation of 3D models are highly dependent on direct manipulation and in many cases offer numerous parameters to adjust the printer settings. Even for sighted users, the complexity of these user interfaces can be rather confusing and impedes its ergonomics.

To simplify the preparation of the 3D model, we identified multiple crucial steps. Some of these steps can be carried out algorithmically, others need feedback from the user. There are already tools that implement some of the following strategies (usually only with a graphical user interface and several interaction steps). According to the author's knowledge, there is currently no approach that is accessible to people who are blind. In addition, there is no approach that combines the entire process chain in such a way that it can be used by people with visual impairments.

The first step of print preparation is the placement of the desired 3D model onto the virtual building plate, which represents the building plate of the 3D printer. With sighted users, this task is usually done by direct manipulation, and thus, is inaccessible for users who are blind. In our approach, the model is always placed in the center of the building plate. To our knowledge, this trivial solution works for almost all 3D models.

Another important task is the definition of the printing orientation of the model. It directly influences the time and (support) material needed to print the model. Since the model has to 

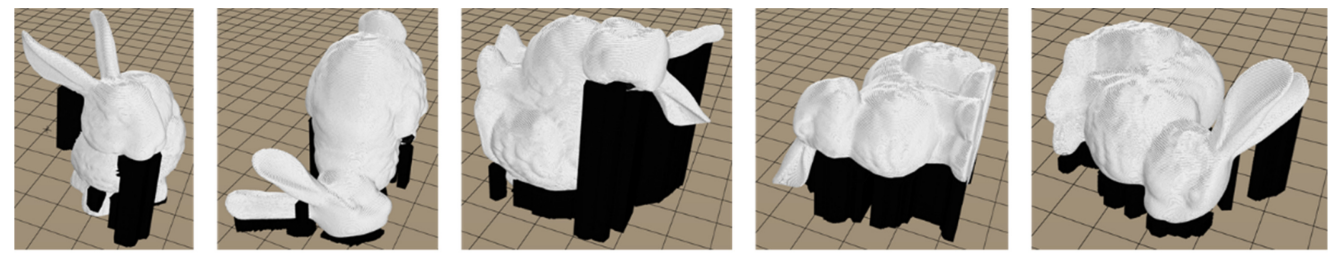

Fig. 4. Different printing directions considerably influence printing costs (black support material), duration, and the quality of adhesion to the building plate.

adhere to the building platform until the printing process finishes, a sufficient bearing area between the platform and the printed model is crucial for the success of the printing process.

A heuristic algorithm can be applied to evaluate which one of multiple orientations is optimal (see Figure 4). In our approach, the model is evaluated from six different directions (both sides of each main axis) regarding multiple parameters. The algorithm chooses the printing orientation to minimize the amount of support material required. This also positively affects the printing duration. If the bearing area is below a threshold value (e.g., a lengthy, vertically oriented object) a better alternative with similar support material requirements is selected instead. If no such alternative can be found, an additional structure of concentric circles consisting of support material is added to the model's base to improve the platform adhesion.

According to the questionnaire, another important parameter is the definition of the printed object's size. Due to the fact that many models are not at scale of their real-world entities (e.g., elephant) it is not constructive to scale the models by a defined factor for printing. Instead, our approach queries the user to define the maximum edge length the object is scaled to. Finally, as an optional parameter to influence the stability of the printed object, the density of the fill structure can be defined by the user. Alternatively, the default setting of $20 \%$ fill density can be used, which is suitable for most 3D models.

\subsection{Transformation into Print Data}

The most common type of consumer 3D printers work according to the fused decomposition modeling. This additive manufacturing technique successively deposits molten printing material (filament) through a print head at defined positions, layer by layer, until the whole 3D object is built. 3D printers are controlled by instructions (so-called $G$-code), which, for example, defines movements of the print head, degree, or the speed of filament extrusion. To transform the 3D model in the form of polygonal meshes into 3D printer instructions, the model has to be subdivided into slices to generate machine readable instructions. This process is usually done by specialized software (slicer). The slicer software needs the source 3D model as well as printing parameters from the user. It also needs knowledge about the target 3D printer's hardware and software (e.g., there are multiple different dialects of G-code that may be implemented in 3D printer firmware).

In our approach, after the user starts the printing process by issuing a voice command, both the selected 3D model and the printing parameters are forwarded to the slicer software. To generate the corresponding G-code for the printer, the details of multiple (dual-head) 3D printers, abstracted in configuration files, can be accessed. After the slicing process, details for printing duration and (support) material consumption can be obtained ${ }^{1}$ and reported to the user.

\footnotetext{
${ }^{1}$ The process of slicing is actually performed six times in sequence to evaluate the optimal printing direction (both sides of each main axis) of the model (see previous step).
} 


\subsection{Printing Process}

Our starting point is that a 3D printer is available on which the print materials have been installed and is ready for operation. Depending on the size of the printed objects, in principle a large number of objects can be created without the help of an assistant.

Autonomous 3D printing for people who are visually impaired makes use of multiple specific features of 3D printers. To obtain optimal results, the printing platform should be occasionally adjusted to the correct distance from the print head. Since this manual task requires differentiated sight, printers that are able to adjust the print platform automatically should be preferred. This automatic leveling of the building plate before each print can be encoded in the G-code, and thus in our 3D printer configuration files.

There is an increasing number of dual-head consumer printers, starting at prices below $\$ 1000$. The second extruder of the print head can be used to print the support structures in a different filament. Since the task of manually removing these supporting structures also requires good vision, a soluble filament should be used for printing the supporting structures. During the printing process, the computed G-code is gradually forwarded to the 3D printer. This allows the user to monitor the progress of the printing process and provides feedback to the user. When the printing process is completed and the heated building plate is cooled down, the user is informed acoustically.

\subsection{Post-Processing of the Printed Model}

Cooling down the heated building plate before the print model is removed has an essential advantage. Due to their differing thermal expansion coefficients, considerable tensions between the building plate and the printed object arise. Hence, the printed object can be removed much easier.

The support structures, necessary to print many 3D models, have to be removed from the 3D model. The removal process of brittle structures is often tedious and can be harmful to the user. In addition, differentiating between the actual model and support structures typically requires discriminating a color difference that may not be possible for VIP. When water-soluble filament is used for the support structures, the users would only have to soak the model in water to remove the support structures. Additionally, for 3D printer platforms that often tightly adhere to the model, an additional bottom layer of soluble material can be printed below the model. In this case, the user only has to remove the building platform from the 3D printer and to rinse it in water to dissolve the support structures. Although it is not necessary for the successful removal of soluble support structures, tepid water accelerates the dissolution.

\section{INITIAL COMPUTER-BASED PROTOTYPE}

This section describes our first implementation of the approach introduced in Section 3. The purpose of this computer-based prototype was to test the principal feasibility of 3D printing for VIP. It achieved the main goals of evaluating the entire process, beginning from the 3D model selection up to the point of obtaining the finished printed objects ready for tactile exploration.

\subsection{Architecture}

The prototypical test environment consisted of a computer and a 3D printer that was connected via a USB cable (see Figure 5). Additionally, to reduce the influence of ambient noise, an external microphone was connected to the computer. The set of 3D models had to be stored in a local directory on the laptop. Furthermore, slicing software was installed on the laptop, whereas a configuration file served to define the characteristics of the $3 \mathrm{D}$ printer. 


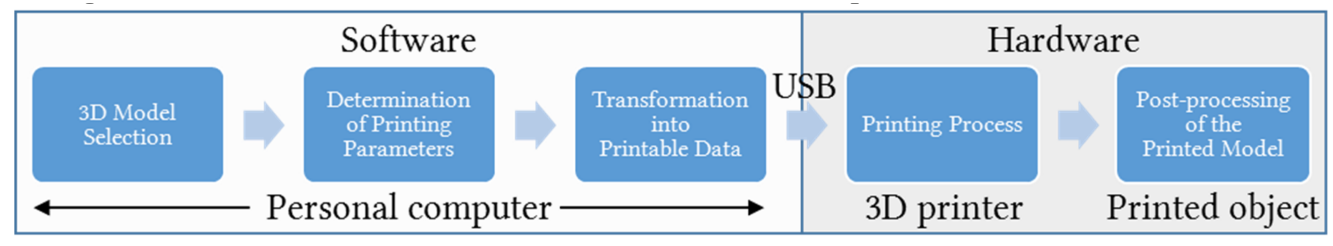

Fig. 5. Test environment of initial prototype.

\subsection{Implementation}

The test environment software was developed as a C\# application. The whole process had to be controlled by voice interaction. For the application's audio feedback, the standard .NET speech synthesis library was used. To make the application controllable by voice commands, the popular voice recognition library PocketSphinx [24] was chosen. This allowed an offline recognition of voice commands and was highly configurable.

This prototype was developed with the objective of testing the whole task, including the actual 3D printing process. To control the duration of the test, in this implementation no parameters were requested from the user to avoid influencing the printing durations. Instead, after selection of a 3D model, the user had to command the computer to start the printing process. This started a process of transforming (slicing) the 3D model into print data. A configurable slicer software CuraEngine [13] generated the machine readable G-code from the 3D model file and the $3 \mathrm{D}$ printer's configuration. Finally, the G-code was sent to the $3 \mathrm{D}$ printer via the USB interface.

\subsection{User Study on Technical Feasibility}

The predominant question for our approach was whether people who are blind are able to use 3D printers when the described adaptations (see Section 3) are implemented. To test this, we performed an initial user study focusing on the technical aspect.

4.3.1 Participants. The user study was carried out in a local educational center for people who are blind or visually impaired. We recruited seven volunteers (five female, two male), whose ages ranged from 16 to 32 years old, with an average of $20.14 \pm 5.4$ years. Three of the participants were completely blind and four possessed only minor residual vision (at least WHO category 3 [9]). None of the participants had experience with 3D printing.

4.3.2 Apparatus. The implementation of the computer-based prototype was installed on a standard notebook (Toshiba Satellite P70-A-104) with an external microphone. For the user study, 3D model candidates were identified and stored in the local directory. Finally, four models were chosen that could be selected by participants using voice recognition. To limit the time needed for each participant to wait during the printing process, these models were manually scaled down to limit their printing duration to less than half an hour.

A dual-head 3D printer (FelixPrinters Felix Pro 1), which supports auto-leveling of the heated building platform, was connected via USB to the notebook. During the printing process, this platform was fixed to the printing base by three integrated electromagnets. When the printing process was finished, these electromagnets released the printing bed so that it could be removed easily. In the experiment, we used a combination of standard PLA (Polylactide) filament for printing the actual model and PVA (Polyvinyl Alcohol) for printing the soluble support structures. 
We carried out the experiment in a lab on the premises of the educational center. The notebook and the 3D printer were placed side by side. A bowl of water was placed on another on the right.

4.3.3 Design and Procedure. The users were tested one at a time. Each of the participants obtained a short briefing from the experimenter on the software interface and the connected 3D printer. Next, they were introduced to the testing procedure and each of the select 3D models were explained. In the briefing, subjects were given the opportunity to ask open questions and to be guided to the microphone, to the 3D printer with its building platform, and to the bowl of water.

After the briefing, the voice recognition was started and the microphone was handed over to the participant. The subjects had to select one of the models in the database by its designation and to confirm their choice. A subsequent voice command initiated the slicing of the 3D model and then the printing process. Dependent on which 3D model was chosen, the printing process required up to 30 minutes. This was reported to the subject at the beginning of the printing process. During the printing process, there was no need for the user to touch the 3D printer. After the print was complete, the subject was asked to wait until the building plate had cooled down and, subsequently, to remove the 3D printed object. Finally, the 3D object had to be placed in the bowl of water to dissolve the support structures.

During the whole test the user was able to ask questions to aid his/her comprehension. The experimenter recorded the time needed to fulfill the task and the requests for help. At the end of the trial, the printed objects were given to the participants as souvenirs.

4.3.4 Results and Discussion. All of the participants successfully accomplished the task to select a 3D model and to start the actual print process. Likewise, they managed to autonomously detach the printed object from the 3D printer's building platform without problems and to dissolve the support structures. On average, the subjects needed $11.14 \pm 3.81$ minutes to accomplish this task (excluding the duration of the actual printing process).

However, multiple technical issues were identified. The main issue was caused by the offline voice recognition, which did not work reliably. Despite our preliminary tests in which it worked without issues, during the experiment multiple subjects had to repeat their commands up to 20 times until the system recognized them correctly. In this case, each of these participants asked the experimenter how they should behave. They were encouraged to continue. This resulted in nonhomogeneous duration requirements for the problem solving, between 7 and 17 minutes. In a retrospective investigation we identified two causes of this shortcoming: the acoustic model implemented was not sufficiently configured and the recognizer module's vocabulary was not optimized for the specific task.

During the experiment, another issue arose because of one of the dual-head 3D printers extruders (PVA filament) stopped printing. Hence, no more water soluble support material could be printed. To stay within the time constraints of the experiment, we decided to continue without an intensive investigation of its cause. Instead, the models were printed using only one extruder. To test the feasibility, the participants were still asked to remove the print from the building plate and to place it in the bowl of water. After the experiment, it became evident that the clogging was caused by a modified configuration of the printing temperatures, i.e., the second extruder's temperature during idle time was too high. Additionally, PVA filament is susceptible to burning in the 3D printer's extruder when it is not used during the print. However, there are PVA filaments that are designed to limit this issue.

Overall, the results of this experiment showed the fundamental feasibility of autonomous 3D printing by people with visual impairments. Based on these results, we speculated about improvements to the user interfaces as well as the technical configuration. 


\section{ADVANCED SMARTPHONE-BASED PROTOTYPE}

This section presents an advanced approach that allows users who are blind to use their smartphones as the user interface to print 3D models. The extended hardware configuration allows the abstraction of the 3D printer's hardware and therefore is more flexible than the first prototype.

\subsection{Architecture}

The computer-based prototype verified the principal feasibility of autonomous 3D printing by people who are blind. However, the proposed architecture had some drawbacks. One major issue was that the computer was tightly coupled with the 3D printer. The user interface and the remaining software components were implemented on the computer. Likewise, the 3D printer's configuration was also defined on the computer, which caused a dependency with the printer. Hence, the user interface had to be used with a specific printer. During the extensive printing process (often several hours), this computer provided users with text-to-speech status updates that were only perceivable when they were in close proximity to the device.

Today, smartphones are now equipped with excellent voice recognition functions and users are often familiar with their use. If the application was installed on a private smartphone, it would also be possible to obtain the auditory status updates about printing progress, regardless of the user's location (assuming there is an internet connection).

Some consumer 3D printers already integrate proprietary Wi-Fi interfaces. However, they are not (yet) unified and some printers do not integrate them at all. Another solution we evaluated was the use of Wi-Fi SD cards (such as the Transcend TS16GWSDHC10), which are common for professional photography. They are designed to offer wireless access to data stored on a host system on which they are regularly mounted (e.g., a photo camera). The intended flow of data is from the SD card to the computer connected to it via Wi-Fi. However, it is also possible to reverse the flow of data $[22,30]$, i.e., to store information on these SD cards mounted in the host device (in our case a 3D printer). This could be used as a generic interface between a smartphone and 3D printers (which normally integrate SD card slots). Using this technique, we were able to remotely store 3D models via Wi-Fi on SD cards mounted in conventional 3D printers. However, to the knowledge of the authors, there is no generic way to control 3D printers by this interface to initiate and control the printing process.

Another issue was the limited computing capacity of smartphones. If they would need to perform the computationally intensive slicing of 3D models, this process would take a long time and would mean a massive expense of response time and the batteries.

Recently, an approach for mobile fabrication [42] was introduced that used a single board computer as an interface between smartphone and 3D printed. This work used this setup for connecting a modified 3D printer for the usage on-the-go. In this article we use a similar setup for a different purpose, i.e., for abstracting 3D printing hardware for a generalized interface to enable access to various 3D printers.

For this purpose we used a credit-card sized single board computer called Raspberry Pi. The current version integrates a quad-core processor and 1GB memory, multiple USB ports and a WiFi adapter for less than $\$ 40$. Peripherals are available, such as a keyboard, a mouse can be connected by USB, a display via HDMI connector. However they are not needed to run this device. The only necessary connection is the micro USB cable from a power supply.

One of the USB ports is physically connected to the 3D printer to control the printing process (see Figure 6). Furthermore, the smartphone that implements the auditory user interface is connected via Wi-Fi to the Raspberry Pi computer. Usually, Linux-based operating systems are used, which allow the integration of manifold software components. In our case, this component integrates the slicer software needed for the transformation into print data. 


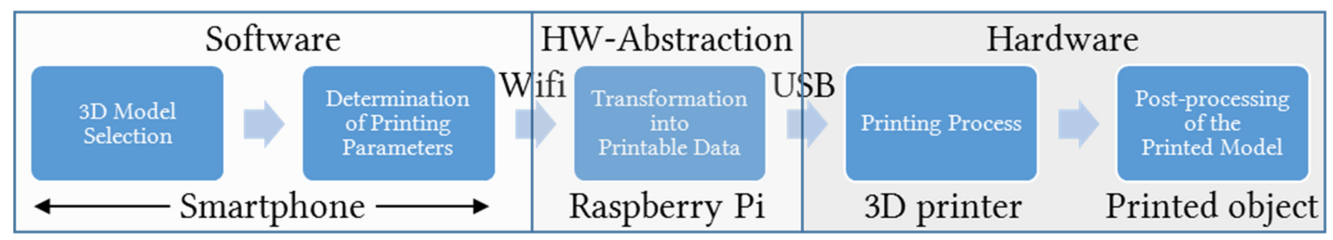

Fig. 6. Test environment of advanced prototype.

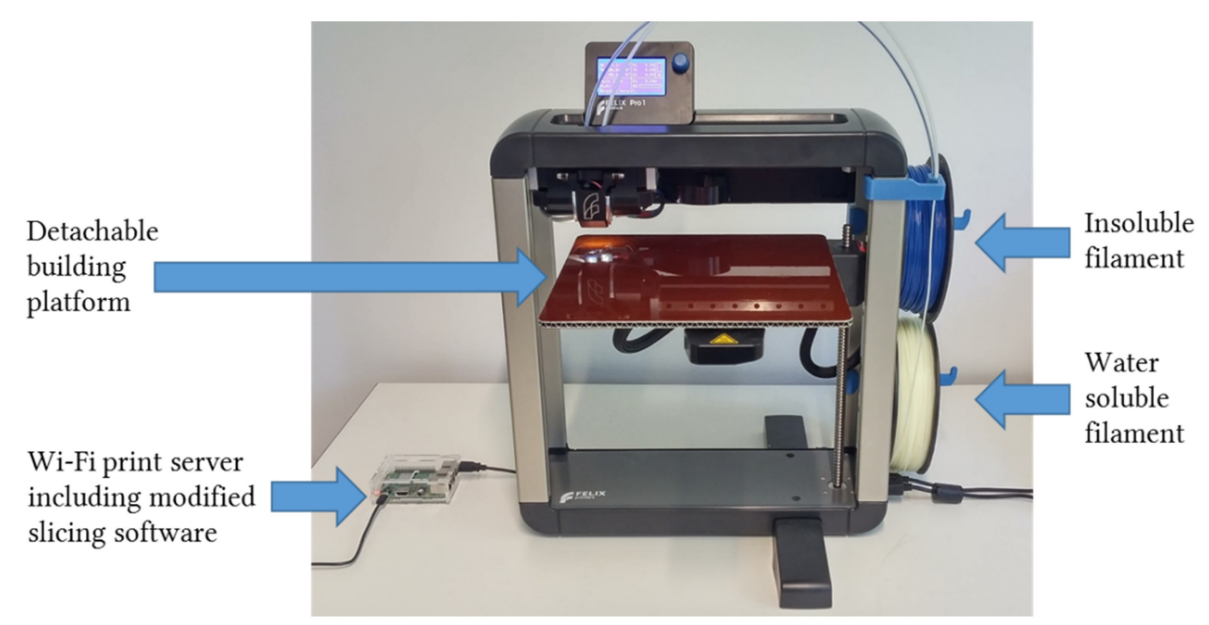

Fig. 7. Combination of Wi-Fi print server and 3D printer connected via USB.

The use of the Raspberry Pi computer as an intermediary interface abstracts all hardwarespecific components of the 3D printer. It works as a 3D print server, which transforms the user's data into machine-specific commands using the 3D printer's configuration data and controls the printer during the printing process. Status updates from the printer (e.g., concerning the printing process) can be forwarded to the user's smartphone, whether the user is nearby or, provided there is internet access. Additionally, in contrast to professional print services, this is advantageous to the user's autonomy, privacy, and incurs only a fraction of the costs.

\subsection{Implementation}

The implementation of the prototype consisted of two units. The mobile application provided the auditory user interface and the print server implemented the hardware abstraction of the 3D printer.

Specialized Print Server. The print server was implemented on a Raspberry Pi 3 (see Figure 7) using a Debian-based Linux operating system. Similar to Reference [42], we used the basic functionality of a software package, OctoPrint [38], which was designed as a web server for 3D printers. For programming purposes, it implemented a REST API [10], which could be used for communication with the mobile application. We had to make adaptions to our server to implement the functionality described in Section 3.2.

The slicer software package CuraEngine [13] has been used to transform the transmitted 3D models of the mobile application into machine readable instructions. The slicing parameters had to be adapted to the needs of dual-head 3D printers. Furthermore, for each incoming 3D model, 


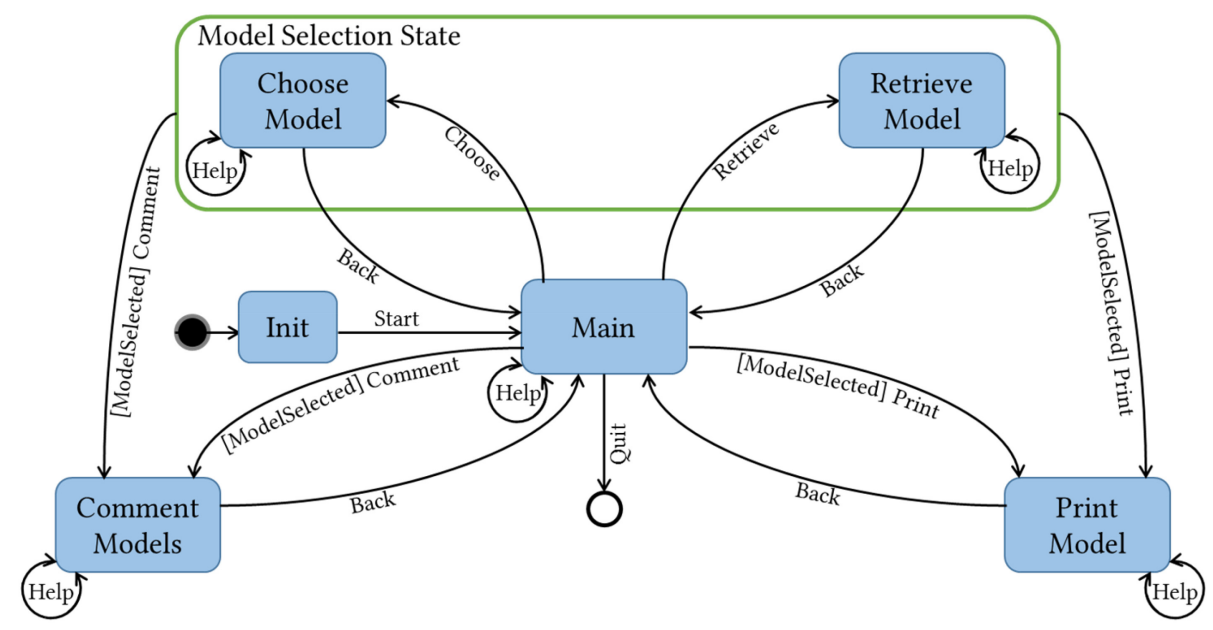

Fig. 8. Simplified state machine for auditory menu structure.

the slicing had to be executed six times (see Section 3) to evaluate the best printing direction and to report the printing duration back to the smartphone application.

Mobile Application. The prototype mobile application was designed for Android smartphones and used the integrated Google speech recognition. The application was designed to allow both the selection of a 3D model from a specialized database of 3D models as well as the option for free 3D model retrieval. We chose the 3D model sharing platform Thingiverse [50] to implement (i) the file storage for our database (including subfolders for the 3D model categories) and (ii) the 3D model retrieval from its extensive database. This platform also used a REST API that could be used for the communication with the smartphone application.

The application itself consisted of four main functionalities. Each of these functionalities were accessible through an auditory menu. To initiate the speech recognition, the user had to tap the display of the smartphone for a short duration and then speak in his or her command.

To enhance the flexibility of the user interface, this menu could be navigated by a state machine (see Figure 8). After starting the application, the users obtained a short description about the structure of the application and which commands could be used. In each state, the help function could be used, which reported the current state and the available options (voice commands) in this state.

It was possible from each of the submenus to navigate back to the main menu. In both of the submenus, Choose model and Retrieve model, the user could select a 3D model. Once a model was selected, the states Comment model and Print model could be accessed directly.

In the state Choose model, one of the predefined categories (technical models, architectural models, nature, persons, art) could be chosen per voice command. In each of these categories, the models could be accessed by the command next, which presented the headlines of the next 10 models to the user. The user was able to obtain a description and comments to a specific model by the command detail, followed by the corresponding number. The command select selected the 3D model which had been explained in detail. The command back navigated back to the Choose model state.

In contrast to Choose model, the state Retrieve model enabled the retrieval of models from the entire 3D model server. By issuing the command search, the application requested the user to define search terms for the query. Subsequently, the retrieval process was started (by using the Thingiverse API) and a resulting set of 3D models was returned to the application. The application 
automatically downloaded the descriptions and file details of the 3D models. To limit the amount of time required to transmit the information, this was restricted to 50 models.

To prevent manual assembly of multiple parts, which usually requires a good visual acuity, the resulting set was filtered. Only 3D models which had exactly one 3D file (STL format) remained in the set. Of the remaining set, the first 10 headlines were reported to the user. The user was able to obtain more information about a specific file by issuing the command detail, followed by the corresponding number. Just as in the state Choose model, the user can select the model which has been explained in detail by the command select. A repeated command search allowed the user to refine the search terms to retrieve more 3D models.

For a selected 3D model, either by Choose model or the state Retrieve model, the state Comment model allowed its annotation with individual explanations of the model or experiences from the printing process. This addressed the willingness of users (see Section 2.4.4) to take an active part in the successive development of a database of user-tested models and the experience of users. If the model was not yet stored in the database of 3D models (see Figure 3) available in the Choose model state, the user was asked in which category this model should be added. If this was not intended, the user was able to navigate back to the main menu. After adding the model to the desired category (or if the model already existed in the database), the user was asked to define a comment. A break ended the recording. Subsequently, the recorded comment was read and had to be approved by the user. The command accept stored the comment along with the 3D model. The command reject navigated back to the main menu.

Finally, the state Print model allowed the user to initiate the print procedure. The user was asked to define the desired size of the model by specifying its maximum edge length. The application confirmed this by repeating the recognized size. This information was used to transfer the 3D model in a reduced size to the print server via the REST API and to slice it to determine a preliminary value for the printing duration. This duration was used to estimate the durations for low, medium, and high-quality 3D printing (defined in the printer configuration; e.g., 8, 15, and 25 micron layer height). These durations were reported to the user, requesting them to define the desired printing quality. Again, the application confirmed the user's choice by repeating the recognized quality and asked the user whether he or she intended to print the model with this configuration. After the user agreed, the 3D model was transferred to the specialized print server and sliced from six orientations to find out the optimal printing orientation. The 3D printing process of the selected candidate was then initiated by the print server, which reported the print progress in percent each minute to their smartphone. When the print was finished and the building platform had cooled down, the users obtained another message on their smartphone.

\subsection{User Study on User Interface}

The intention of this user study was to test whether the users were able to retrieve and to select $3 \mathrm{D}$ models from the internet and to initiate the actual print process.

5.3.1 Participants. The user study was conducted with 13 participants (all male). Their ages ranged from 28 to 55 years, with an average of $42.08 \pm 8.58$ years. They were recruited from a local vocational training center for people with visual impairments on a voluntary basis. Nine of the participants reported being blind (WHO category 4/5 [9]), three more reported a residual vision of $\leq 5 \%$ (WHO category 3 [9]), and one participant had a residual vision of $7 \%$ (WHO category 2 [9]). Three participants were congenitally blind, the remaining entered severe visual impairment (at least WHO category 2 [9]) at an age greater than 18 years.

Most of the participants $(n=11)$ had experience with voice control using smartphones. However, only two participants worked with 3D models in the past (i.e., had at least once loaded a 
specific 3D model into a computer program). Another two participants remarked that 3D models were present in games they played on the computer years ago when they still had sufficient residual vision. None of the participants reported any experience with 3D printing.

5.3.2 Apparatus. This user study was carried out with an off-the-shelf smartphone LG G Flex2. The installed operating system was Android 5.1.1 and was equipped with the standard text-tospeech engine, including language packs for multiple languages. An implementation as described in Section 5.2 was installed on this device. Additionally, this application recorded the timings of the user interactions. The states for retrieving a model and to print a 3D model were implemented as described. To limit the time needed for the user study, the search dialog allowed the selection of one of the models of the retrieval's resulting set, but only read the headline instead of the whole model description. Finally one of these models could be selected for the print process.

Unlike in the initial user study, the actual 3D printing process was not directly carried out. Instead, the 3D models were transferred to the print server. It consisted of a Raspberry Pi 3 with installed Debian Linux operating system, a modified version of the OctoPrint software (see Section 5.2), the slicer software, and a configuration file for the 3D printers (see Section 4.3.2).

5.3.3 Design and Procedure. This user study was designed to verify that people who are blind can retrieve 3D models from the internet by voice commands through the implemented mobile application and to print a selected model using the proposed Wi-Fi interface with the print server. To the knowledge of the authors (concordantly with References $[6,7]$ ) there is no accessible tool for 3D model processing or printing. We did not design our study with a control group because, without such a tool, the unassisted selection and printing of 3D models in comparable settings is not currently possible for people who are blind.

At the beginning of the test, the experimenter briefly explained the purpose of the mobile application, presented multiple examples of printed 3D models, and answered questions. He explained the application's structure and its four submenus. The function of the submenus were explained and that the help command can be used in each menu, which explains the available voice commands. Next, it was explained that only the options search and print are relevant in this user study. To initiate a voice command, the users had to tap on the display of the smartphone.

The user's task was to search for a 3D model of their choice by 3D model retrieval and to select one of the models in the list of retrieved models. Next, they had to navigate to the print menu and specify the size of the model. After the application reported the time needed for printing the model in this size, the user had to specify the print quality (low, medium, high) the model has to be printed in. If the desired quality was not medium, the updated printing time was reported. Thereafter, the printing process started.

During the test, the participants had the chance to ask the experimenter for help. When the user had no idea of an suitable value or search term, the experimenter suggested an exemplary search term. After the test, the users were asked about their impressions and requested to suggest improvements.

5.3.4 Results. Each of the participants was able to retrieve a 3D model and to prepare it for the printing process. Six of the participants had no idea of what to use as search term, so the experimenter suggested an exemplary search term and encouraged the participant to use a similar (but different) term. Used terms of the users were (translated): Eiffel tower, pyramid, switch, eagle owl, wheel rim, humerus, diesel, elephant, rat, dog, apple, paraboloid.

On average, each user initiated $2.46 \pm 1.6$ search queries before selecting a model to print, whereas each query resulted in $2.38 \pm 3.1$ results (see Appendix B). On average, each user spent $128 \pm 62.65$ seconds on the retrieval until selecting a model to print. 
The users needed $49.69 \pm 26.31$ seconds on average to define the print parameters before the 3D model was transferred to the 3D model print server. For the size, they chose on average a maximum edge length size of $8.85 \pm 2.77 \mathrm{~cm}$ and the desired quality was almost uniformly distributed (low: 4; medium: 5; high: 4).

All participants were very pleased with the opportunity to find and print 3D models on their own. Several of them were overwhelmed by the variety of searchable models, and, as some commented, at times strained. They said it might help to listen to someone experienced in the search.

A point of criticism from several participants concerned the multilingual input and output, since most models were only available in their English descriptions. The participants often searched for models in their native language, whereas most 3D models were present in English. This sometimes led to few or no search results. When the participants used the English translation of their search terms this led to more results.

Multiple users reported that the speed of the text-to-speech output was much too slow. They suggested there should be a setting for the speed of speech output. One of the participants proposed the integration of an additional functionality to define exact search terms which are not common in natural language. Another idea was that it might be advantageous to add a visual menu with simple keys to the voice control.

5.3.5 Discussion. Some participants had obvious difficulty with the variety of available models. We assume that one reason for this could be the cross-media search. That is, even though the use of text-retrieval search engines is widely known, it may not be intuitive that text-based searches actually produce 3D models (and their descriptions) as results. However, we assume that this is not specific to VIP. As pointed out by several subjects, it might help to listen to an experienced user. A listening tutorial might be similarly useful.

The reported issue of multilingual input (only for non-English speaking persons) could be resolved in future versions by cross-language information retrieval (e.g., References [18, 35]). In some cases, the search results included models with headlines in multiple languages. A better solution would have been a language detection functionality on the description to dynamically select a text-to-speech engine of the corresponding language. Because foreign languages could still cause confusion to some users, an automated translation service could be integrated.

Another issue was the question about the desired size, i.e., the "maximum edge length." Each of the users asked about the meaning of this phrase. The experimenter explained the fact that the model is uniformly scaled into a box of the edge length of the defined value. However, there is evidence [28] that measurement is an inherently uncertain process. Since the available models are geometrically very different 3D models and from various domains, it could be very difficult for people who are blind to imagine a certain size of an object. This applies in particular if they have never touched such an object before. For this reason, we suggest that users add annotations to 3D models that have already been printed, and share their experiences about features such as optimum print size, among others.

As explained in the introduction, this article focuses on printing 3D models for tactile exploration. However, for functional models such as a door lever, trivial scaling of the model would not be sufficient. According to Reference [28], in the future, automated tools could be developed which generate modular components which do not lose their functionality by scaling. This could be useful for people who are blind.

As stated in Section 3, we chose to integrate voice recognition instead of typing text. To exclude secondary effects caused by residual vision, the prototypical implementation of the interaction in the user study consisted solely of voice control. Although speech recognition worked surprisingly reliably, it sometimes made it more difficult to interact and search. As suggested by several 
participants, a visual menu could be used in the future as a supplement to voice commands. In this way, normal screen-readers could be used with the smartphone application. Such an implementation could be realized by dynamically generating buttons for all possible state transitions of the current state.

To overcome the issue of entering complicated search terms, a normal text input could be integrated. In order not to exclude children, the voice recognition could be used to spell complicated search terms.

\section{CONCLUSIONS}

This article presented several contributions to the practical feasibility of a tool for the autonomous search for and printing of 3D models by people who are blind. It conducted a survey that addressed questions about the requirements and design of such a tool. We provided a workflow for this task, identified target group-specific difficulties in the individual steps, and presented possible solutions. We developed an initial prototype that showed the principal feasibility of 3D printing by VIP in a dedicated experiment. Based on this, we developed an architecture that abstracts the hardwarespecific details of 3D printing into a dedicated component and implemented it as a generic 3D printing interface for people with visual impairments. A second implementation realized a mobile application serving as the user interface to implement a state machine for speech interaction. Finally, a user study was carried out that evaluated its usability and showed further directions of research in this area. The focus of this work was on the complex task of demonstrating the feasibility of the entire workflow. Further studies will focus on developing more sophisticated implementations for the individual subproblems such as improving search results or to control the tactile complexity of retrieved 3D models.

In the future, the annotated database used to share previously printed 3D models and the users' experiences could also be used as a platform for the exchange of teaching resources between educational institutions for people with visual impairments. The lack of reliability and consistent printing quality of 3D printers still impedes the success of practical applicability of 3D printers and has already been addressed by other researchers [7]. Because there is a considerable mass market for consumer-grade 3D printers driving this technology, the authors suppose that product quality and reliability will considerably improve over the next few years. Our approach implemented strategies to reduce the chance of poor-quality print results (see Section 3). Due to the increased accessibility to people with visual impairments for searching and printing 3D models, this could help to raise the attention of the 3D printer market to this group.

By adding an automatically generated visual menu (see Section 5.3.5), this application could also be used by sighted people. Because it considerably eases the handling of 3D models and their preparation for 3D printing, it could be of interest to people who attach more importance to the printed object than the actual printing process. With regard to Design for All, this could help to reduce the inhibition threshold for the active use of 3D printing.

Up to now, consumer 3D printers are only partially reliable. Although there are already products that print many models reliably after a basic setting, without the need for any readjustment. Nevertheless, situations can occur that deviate from the normal situation. For such cases, crowdsourcing techniques (such as VizWiz [4]) could be used to quickly receive feedback from experts and to resolve the situation. Another application would be the use of crowdsourcing to find the right model. One possible solution for the future would be to ask sighted people questions about the visualization of 3D models in a search result set via smartphone.

In the future, the comments of the annotated database could be analyzed to assess the quality of search results. To facilitate this, users could be encouraged to add both positive and negative comments to the models retrieved. Another question that could be covered is the actual understanding 
of the spatial facts through 3D models. Of particular interest is what, in addition to palpability, are the determinants for a good understanding of 3D objects.

Finally, multiple works already addressed the issues people with visual impairments have when using existing search engines (e.g., References [2, 33, 43]) to browse HTML web pages. Other approaches address web page retrieval for other groups of people with special needs such as children (e.g., Reference [25]), elderly people (e.g., Reference [1]), people with dyslexia (e.g., References $[3,36])$, and image retrieval tailored for people with color vision impairment (e.g., Reference [31]). Another interesting research question is specialized 3D retrieval engines for VIP. These could integrate analytic functions (e.g., degree of manifoldness, roundness, size of individual geometric features) similar to those that are used for shape-driven 3D retrieval engines (e.g., Reference [46]) to find out which of the models are good candidates for tactile exploration. For too complex models, specialized simplification algorithms could be used to reduce tactile complexity.

\section{APPENDIX A}

QUESTIONAIRE (translated)

Age

Sex

Visual acuity

Age of entering severe visual impairment

Experience with voice control

Experience with 3D models

(1) How many 3D models have you used so far for tactile exploration (e.g., tactile models at school, building models, animal or machine models)? (In this question there are no approximate flat models meant.)
a. $0-10$
b. $11-50$
c. $51-100$
d. More

(2) Thousands of 3D models are available free of charge on the internet. Most of these models can be printed with $3 D$ printers, which makes them suitable for tactile exploration. Would it be desirable if these models could be printed at home?
a. Yes
b. No

(3) Would it be advantageous if the selection and printing of 3D models could be done autonomously? I.e., without a (printing) company or a seeing assistant?
a. Yes
b. No

(4) Suitable 3D printers are currently available for about 800 Euros. Could you imagine buying such a printer to print $3 D$ models on your own?

a. Yes

b. No

(5) In which areas are you interested in 3D models? (Multiple answers are possible in this question.)

a. Technical models (e.g., machines, ...)

b. Architectural models (e.g., buildings, ...)

c. Art (e.g., sculptures, ...) 
d. Nature (e.g., plants, ...)

e. Persons (e.g., faces, ...)

Please write down further ideas:

(6) Suppose there was a smartphone application for retrieving and printing 3D models-would you install it?

a. Yes

b. No

(7) If so, would you (actively) use a feature to discuss and share your experiences with others about already $3 D$ models printed?

a. Yes

b. No

(8) What print settings should be provided by a smartphone application? For each of the following four questions it is allowed to tick an option. These options are: Very Unimportant, Slightly Unimportant, Neutral, Slightly Important, Very Important.

a. Printing Size

i. Very Unimportant

ii. Slightly Unimportant

iii. Neutral

iv. Slightly Important

v. Very Important

b. Settings for Printing Quality

i. Very Unimportant

ii. Slightly Unimportant

iii. Neutral

iv. Slightly Important

v. Very Important

c. Settings for Printing Duration

i. Very Unimportant

ii. Slightly Unimportant

iii. Neutral

iv. Slightly Important

v. Very Important

d. Settings for the Stability of Prints
$i$. Very Unimportant
ii. Slightly Unimportant
iii. Neutral
iv. Slightly Important
v. Very Important

Please write down further ideas:

(9) Please indicate some general information

a. Age:

b. Degree of Visual Impairment in Percent (if blind, please write down 0\%):

(10) If you are interested in being available as a test user for a smartphone application in the future, please indicate your e-mail address: 


\section{APPENDIX B}

Exemplary 3D models retrieved by visually impaired people in second user study.

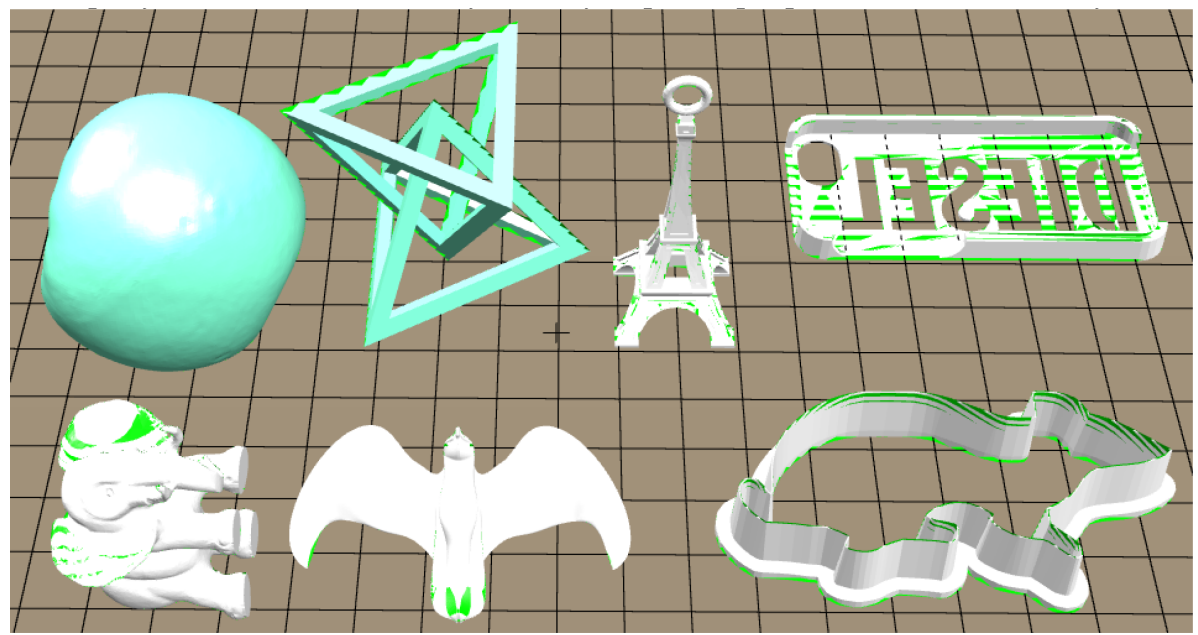

\section{APPENDIX C}

Further exemplary 3D models printed with soluble support structures (white elements).

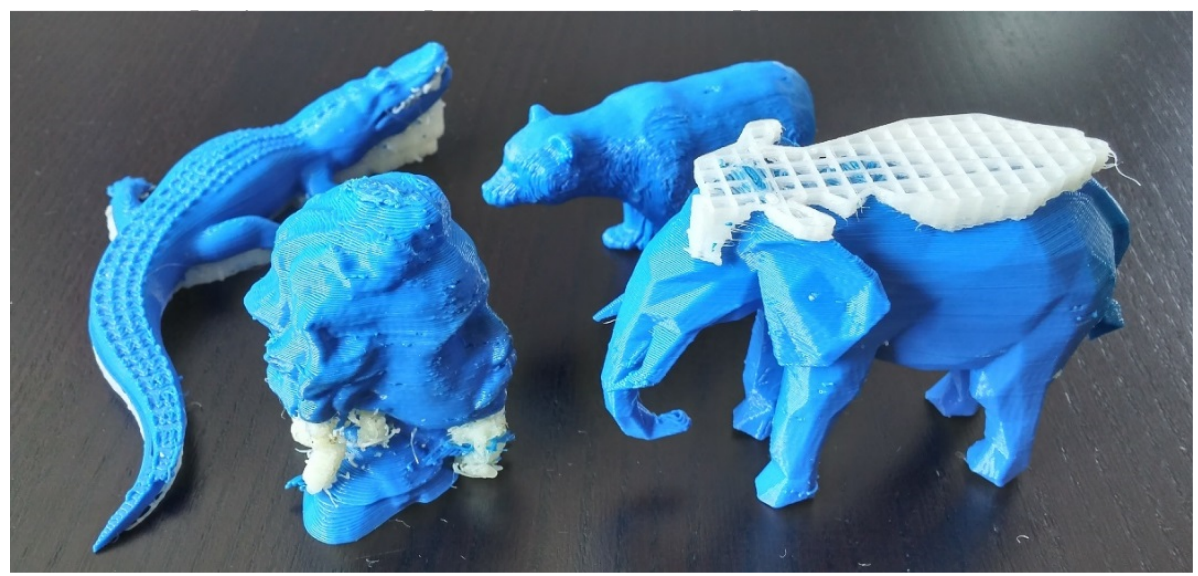

\section{ACKNOWLEDGMENTS}

We would like to thank the Vocational Training Centre for the Blind and Visually Impaired (BFW Würzburg) and the Educational Center for Blind and Visually Impaired People (BBS Nürnberg) as well as everyone involved in both user studies and the questionnaire.

\section{REFERENCES}

[1] S. Anderson, N. Liberman, E. Bernstein, S. Foster, E. Cate, B. Levin, and R. Hudson. 1999. Recognition of elderly speech and voice-driven document retrieval. Proceedings of the IEEE International Conference on Acoustics, Speech, and Signal Processing (1999). IEEE, 145-148.

[2] P. Andronico, M. Buzzi, C. Castillo, and B. Leporini. 2006. Improving search engine interfaces for blind users: A case study. Universal Access in the Information Society 5, 1 (2006), 23-40. 
[3] G. Berget and F. E. Sandnes. 2016. Do autocomplete functions reduce the impact of dyslexia on information-searching behavior? The case of Google. Journal of the Association for Information Science and Technology 67, 10 (Oct. 2016), 2320-2328. DOI:https://doi.org/10.1002/asi.23572

[4] Jeffrey P. Bigham, Chandrika Jayant, Hanjie Ji, Greg Little, Andrew Miller, Robert C. Miller, Robin Miller, Aubrey Tatarowicz, Brandyn White, Samual White, and Tom Yeh. 2010. VizWiz: Nearly real-time answers to visual questions. In Proceedings of the 23rd Annual ACM Symposium on User Interface Software and Technology. ACM, 333-342.

[5] C. Brown and A. Hurst. 2012. VizTouch: Automatically generated tactile visualizations of coordinate spaces. Proceedings of the 6th International Conference on Tangible, Embedded and Embodied Interaction (2012). ACM, 131-138.

[6] E. Buehler, S. K. Kane, and A. Hurst. 2014. ABC and 3D: Opportunities and obstacles to 3D printing in special education environments. Proceedings of the 16th International ACM SIGACCESS Conference on Computers \& Accessibility (2014). ACM, 107-114.

[7] Erin Buehler, Niara Comrie, Megan Hofmann, Samantha McDonald, and Amy Hurst. 2016. Investigating the implications of 3D printing in special education. ACM Transactions on Accessible Computing 8, 3 (Mar. 2016), 1-28. DOI:https://doi.org/10.1145/2870640

[8] Erin Buehler, Stacy Branham, Abdullah Ali, Jeremy J. Chang, Megan Kelly Hofmann, Amy Hurst, and Shaun K. Kane. 2015. Sharing is caring: Assistive technology designs on Thingiverse. In Proceedings of the 33rd Annual ACM Conference on Human Factors in Computing Systems. ACM, 525-534.

[9] Change the Definition of Blindness: 2017. http://www.who.int/blindness/Change\%20the\%20Definition\%20of\%20 Blindness.pdf?ua=1. Accessed: 2017-04-04.

[10] R. Fielding. 2000. Representational state transfer. Architectural Styles and the Design of Network-Based Software Architecture (2000), 76-85.

[11] E. Folmer, B. Yuan, D. Carr, and M. Sapr. 2009. TextSL: A command-based virtual world interface for the visually impaired. Proceedings of the 11th International ACM SIGACCESS Conference on Computers and Accessibility (2009). ACM, 59-66.

[12] Thomas Funkhouser, Patrick Min, Michael Kazhdan, Joyce Chen, Alex Halderman, David Dobkin, and David Jacobs. 2003. A search engine for 3D models. ACM Transactions on Graphics 22, 1 (2003), 83-105.

[13] GitHub. Ultimaker/CuraEngine. Retrieved June 6, 2017 from https://github.com/Ultimaker/CuraEngine.

[14] S. Goose and C. Möller. 1999. A 3D audio only interactive web browser: Using spatialization to convey hypermedia document structure. Proceedings of the 7th ACM International Conference on Multimedia (Part 1) (1999). ACM, 363-371.

[15] T. Götzelmann, Lisa Branz, Claudia Heidenreich, and Markus Otto. 2017. A personal computer-based approach for 3D printing accessible to blind people (short paper). Proceedings of the 10th International Conference on PErvasive Technologies Related to Assistive Environments (PETRAE'17). ACM.

[16] T. Götzelmann. 2016. LucentMaps: 3D printed audiovisual tactile maps for blind and visually impaired people. Proceedings of the 18th International ACM SIGACCESS Conference on Computers and Accessibility (ASSETS'16). ACM.

[17] T. Götzelmann and A. Pavkovic. 2014. Towards automatically generated tactile detail maps by 3D printers for blind persons. Proceedings of the 14th International Conference on Computers Helping People with Special Needs (ICCHP'14). Springer, $1-7$.

[18] G. Grefenstette. 1998. Cross-Language Information Retrieval. Kluwer, Dordrecht.

[19] J. Gual, M. Puyuelo, and J. Lloveras. 2015. The effect of volumetric (3D) tactile symbols within inclusive tactile maps. Applied Ergonomics 48 (May 2015), 1-10. DOI:https://doi.org/10.1016/j.apergo. 2014.10.018.

[20] Anhong Guo, Jeeeun Kim, Xiang 'Anthony' Chen, Tom Yeh, Scott E. Hudson, Jennifer Mankoff, and Jeffrey P. Bigham. 2017. Facade: Auto-generating tactile interfaces to appliances. ACM, 5826-5838.

[21] S. Hackett, B. Parmanto, and X. Zeng. 2004. Accessibility of internet websites through time. ACM SIGACCESS Accessibility and Computing. ACM, 32-39.

[22] Hackaday. Hacking Transcend Wifi SD Cards. Retrieved February 3, 2017 from http://hackaday.com/2013/08/12/ hacking-transcend-wifi-sd-cards.

[23] Yahoo! Japan. Hands On Search. Retrieved February 3, 2017 http://sawareru.jp/en.

[24] D. Huggins-Daines, M. Kumar, A. Chan, A. W. Black, M. Ravishankar, and A. I. Rudnicky. 2006. Pocketsphinx: A free, real-time continuous speech recognition system for hand-held devices. In Proceedings of IEEE International Conference on Acoustics, Speech and Signal Processing, vol. 1. IEEE, I-I .

[25] Michel Jansen, Wim Bos, Paul van der Vet, Theo Huibers, and Djoerd Hiemstra. 2010. TeddIR: Tangible information retrieval for children. Proceedings of the 9th International Conference on Interaction Design and Children. ACM, $282-285$.

[26] N. Kaklanis, D. Tzovaras, and K. Moustakas. 2009. Haptic navigation in the world wide web. Universal Access in Human-Computer Interaction. Applications and Services, C. Stephanidis (Ed.). Springer, Berlin, 707-715.

[27] S. K. Kane and J. P. Bigham. 2014. Tracking @stemxcomet: Teaching programming to blind students via 3D printing, crisis management, and Twitter. In Proceedings of the 45th ACM Technical Symposium on Computer Science Education ACM, 247-252. 
[28] Jeeeun Kim, Anhong Guo, Tom Yeh, Scott E. Hudson, and Jennifer Mankoff. 2017. Understanding uncertainty in measurement and accommodating its impact in 3D modeling and printing. In Proceedings of the 2017 Conference on Designing Interactive Systems. ACM, 1067-1078.

[29] J. Kim and T. Yeh. 2015. Toward 3D-printed movable tactile pictures for children with visual impairments. Proceedings of the 33rd Annual ACM Conference on Human Factors in Computing Systems (Seoul, Republic of Korea, 2015). ACM, 2815-2824.

[30] S. Konstantaras and C. Dillon. 2014. Offensive Technologies Project Report: Exploiting Vulnerabilities of Wi-Fi SD cards. University of Amsterdam. https://www.os3.nl/_media/2013-2014/courses/ot/connor_stavros.pdf. (Accessed: 2017-04$04)$.

[31] V. A. Kovalev. 2004. Towards image retrieval for eight percent of color-blind men. Proceedings of the 17th International Conference on Pattern Recognition (ICPR'04). IEEE, 943-946.

[32] Richard E. Ladner, Melody Y. Ivory, Rajesh Rao, Sheryl Burgstahler, Dan Comden, Sangyun Hahn, Matthew Renzelmann, Satria Krisnandi, Mahalakshmi Ramasamy, Beverly Slabosky, Andrew Martin, Amelia Lacenski, Stuart Olsen, and Dmitri Groce. 2005. Automating tactile graphics translation. Proceedings of the 7th International ACM SIGACCESS Conference on Computers and Accessibility (2005). ACM, 150-157.

[33] B. Leporini, P. Andronico, and M. Buzzi. 2004. Designing search engine user interfaces for the visually impaired. Proceedings of the 2004 International Cross-disciplinary Workshop on Web Accessibility (W4A'04). ACM, 57-66.

[34] S. Leuthold, J. A. Bargas-Avila, and K. Opwis. 2008. Beyond web content accessibility guidelines: Design of enhanced text user interfaces for blind internet users. International fournal of Human-Computer Studies 66, 4 (Apr. 2008), 257270. DOI:https://doi.org/10.1016/j.ijhcs.2007.10.006

[35] M. L. Littman, S. T. Dumais, and T. K. Landauer. 1998. Automatic cross-language information retrieval using latent semantic indexing. Cross-Language Information Retrieval, G. Grefenstette (Ed.). The Springer International Series on Information Retrieval, vol. 2. Springer, Boston, MA, 51-62.

[36] A. MacFarlane, A. Al-Wabil, C. R. Marshall, A. Albrair, S. A. Jones, and P. Zaphiris. 2010. The effect of dyslexia on information retrieval: A pilot study. Fournal of Documentation 66, 3 (Apr. 2010), 307-326. DOI:https://doi.org/10.1108/ 00220411011038421

[37] V. Nemec, Z. Mikovec, and P. Slavik. 2003. Adaptive navigation of visually impaired users in a virtual environment on the world wide web. Universal Access Theoretical Perspectives, Practice, and Experience: 7th ERCIM International Workshop on User Interfaces for All, N. Carbonell and C. Stephanidis (Eds.). Springer, Berlin, 68-79.

[38] OctoPrint. OctoPring.org. Retrieved June 13, 2017 from http://octoprint.org.

[39] H. Petrie, F. Hamilton, and N. King. 2004. Tension, what tension?: Website accessibility and visual design. Proceedings of the 2004 International Cross-Disciplinary Workshop on Web Accessibility (W4'04). ACM, 13-18.

[40] L. S. Petrucci, E. Harth, P. Roth, A. Assimacopoulos, and T. Pun. 2000. WebSound: A generic web sonification tool, and its application to an auditory Web browser for blind and visually impaired users. Proceedings of the 6th International Conference on Auditory Display (ICAD’00).

[41] Patrick Roth, Lori Petrucci, Thierry Pun, and André Assimacopoulos. 1999. Auditory browser for blind and visually impaired users. Extended Abstracts on Human Factors in Computing Systems (CHI'99). ACM, 218-219.

[42] T. Roumen, B. Kruck, T. Dürschmid, T. Nack, and P. Baudisch. 2016. Mobile fabrication. In Proceedings of the 29th Annual Symposium on User Interface Software and Technology. ACM, 3-14.

[43] F. Seyedarabi and R. Calvo. 2016. Search engines: New widgets, new accessibility challenges. In Proceedings of the 7th International Conference on Software Development and Technologies for Enhancing Accessibility and Fighting InfoExclusion. ACM, 54-61.

[44] L. Shi, I. Zelzer, C. Feng, and S. Azenkot. 2016. Tickers and talker: An accessible labeling toolkit for 3D printed models. In Proceedings of the 2016 CHI Conference on Human Factors in Computing Systems. ACM, 4896-4907.

[45] Sketchfab. Your 3D content on web, mobile, AR, and VR. Retrieved January 4, 2018 from https://sketchfab.com.

[46] H. Sundar, D. Silver, N. Gagvani, and S. Dickinson. 2003. Skeleton based shape matching and retrieval. Shape Modeling International, 2003. IEEE, 130-139.

[47] Saiganesh Swaminathan, Thijs Roumen, Robert Kovacs, David Stangl, Stefanie Mueller, and Patrick Baudisch. 2016. Linespace: A sensemaking platform for the blind. Proceedings SIGCHI Conf. Human Factors in Computing Systems. ACM.

[48] N. Takagi and J. Chen. 2014. Development of a computer-aided system for automating production of tactile maps and its usability evaluation. World Automation Congress (WAC'14). IEEE, 213-218.

[49] B. Taylor, A. Dey, D. Siewiorek, and A. Smailagic. 2016. Customizable 3D printed tactile maps as interactive overlays. ACM, 71-79.

[50] Thingiverse. Digital Designs for Physical Objects. Retrieved from January 4, 2018. https://www.thingiverse.com.

[51] Z. Wang, B. Li, T. Hedgpeth, and T. Haven. 2009. Instant tactile-audio map: Enabling access to digital maps for people with visual impairment. Proceedings of the 11th International ACM SIGACCESS Conference on Computers \& Accessibility (2009). ACM, 43-50. 
[52] T. Watanabe, T. Yamaguchi, K. Watanabe, J. Akiyama, K. Minatani, M. Miyagi, and S. Oouchi. 2011. Development and evaluation of a tactile map automated creation system accessible to blind persons. The Transactions of the Institute of Electronics, Information and Communication Engineering D J 94-D, 10 (Oct. 2011), 1652-1663.

[53] Tetsuya Watanabe, Kosuke Araki, Toshimitsu Yamaguchi, and Kazunori Minatani. 2016. Development of tactile graph generation web application using R statistics software environment. IEICE Transactions on Information and Systems E99.D 8 (2016), 2151-2160. D0I:https://doi.org/10.1587/transinf.2015EDP7405

[54] T. P. Way and K. E. Barner. 1997. Automatic visual to tactile translation. II. evaluation of the TACTile image creation system. IEEE Transactions on Rehabilitation Engineering 5, 1 (1997), 95-105.

[55] G. R. White, G. Fitzpatrick, and G. McAllister. 2008. Toward accessible 3D virtual environments for the blind and visually impaired. Proceedings of the 3rd International Conference on Digital Interactive Media in Entertainment and Arts (2008). ACM, 134-141.

[56] Yobi3D. Free 3D Models Search Engine. Retrieved January 4, 2018 from http://www.yobi3d.com.

[57] W. Yu, R. Kuber, E. Murphy, P. Strain, and G. McAllister. 2006. A novel multimodal interface for improving visually impaired people's web accessibility. Virtual Reality 9, 2-3 (Jan. 2006), 133-148. DOI:https://doi.org/10.1007/ s10055-005-0009-z.

[58] M. Zajicek, C. Powell, and C. Reeves. 1998. A web navigation tool for the blind. Proceedings of the 3rd International ACM Conference on Assistive Technologies (1998). ACM, 204-206.

Received June 2017; revised May 2018; accepted July 2018 